\title{
Pseudo-differential Operators and Deformation Quantization
}

\author{
B.Fedosov \\ University of Potsdam
}

\begin{abstract}
Using the Riemannian connection on a compact manifold $X$, we show that the algebra of classical pseudo-differential operators on $X$ generates a canonical deformation quantization on the cotangent manifold $T^{*} X$. The corresponding Abelian connection is calculated explicitly in terms of the of the exponential mapping. We prove also that the index theorem for elliptic operators may be obtained as a consequence of the index theorem for deformation quantization.
\end{abstract}

\section{Introduction}

It is well known that the algebra of pseudo-differential operators ( $\psi$ do) on a manifold $X$ may be viewed as a quantization of the algebra of their leading symbols. The corresponding symplectic manifold (phase space) is $M=T^{*} X$ with a standard symplectic form

$$
\omega=d q^{i} \wedge d p_{i}, \quad i=1,2, \ldots, n=\operatorname{dim} X .
$$

However, the quantization procedure allowing one to construct a $\psi$ do with a given leading symbol is by no means canonical. Usually it is based on a partition of unity subordinate to a coordinate covering of $X$ and at the first glance has nothing to do with the canonical construction of deformation quantization for general symplectic manifolds [3, 4]. Many times I was asked by my colleagues about the meaning of the canonical deformation quantization of $[3,4]$ for the case of $\psi$ do but was not able to give a satisfactory 
answer. So, I decided to study this question more carefully. As a result this paper appeared.

We use a well-known idea that the linear connection on $X$ enables one to construct an invariant calculus of psido. This idea was suggested first in [1] and was emploied later many times in different situations (see, e.g. $[9,8,7]$ ). Deformation quantization on cotangent bundles and its relation to psido was also intensively studied by M. Bordemann with collaborators and M. Pflaum, see, e.g., [2] and references therein.

Another question discussed in the paper is a connection between the Atiyah-Singer index theorem for elliptic $\psi$ do and the index theorem for deformation quantization. A simple comparison shows that the former is a particular case of the latter, so we can conclude a posteriori that the AtiyahSinger theorem follows from the index theorem for deformation quantization. And again, I was asked many times if such a conclusion may be done a priori. As expected, the answer is positive.

Finally, the third question discussed here is how to pass from a special form of the index theorem for deformation quantization to the general one. By a special form we understand an explicit formula for $\operatorname{Tr} 1$ in the case of a compact symplectic manifold $M$. This is an essential step because the cotangent bundle $M=T^{*} X$ is never compact.

Now, gethering everything together, we get a strategy to derive the AtiyahSinger index theorem from the index theorem for deformation quantization. We start with the algebra of $\psi \mathrm{do}$, then pass to the algebra of formal symbols and the algebraic index formula for them (Section 1), then pass to a canonical deformation quantization on $M=T^{*} X$ and show that this canonical procedure yields the algebra of formal symbols (Section 2). Next, we show that the index theorem for deformation quantization on any symplectic manifold $M$ (and thus on $M=T^{*} X$ ) follows from an explicit formula for $\operatorname{Tr} 1$ on a compact symplectic manifold $M$ (Section 3 ). This is done using the Atiyah-Bott-Patodi method in deformation quantization [6].

Acknowledgments. The author would like to thank those of his colleagues whose questions showed the necessity of writing the present paper. Especially I am grateful to Professor N. Tarkhanov whose questions were the most persistent ones.

This article was written during my stay at the Department of Mathematics, Royal Institute of Technology, Stockholm. I would like to thank Professor A. Laptev, the chief of the Department, for invitation and hospitality. 


\section{The Algebra of Formal Symbols}

We recall briefly the notion of formal symbols and their application to the index theorem for elliptic pseudo-differential operators. The proofs are mostly omitted and can be found in [5].

Let $E^{0}, E^{1}$ be complex vector bundles of ranks $m^{0}, m^{1}$ over a manifold $X$. We define a space $\mathcal{A}=\mathcal{A}(X)=\mathcal{A}\left(X, E^{0}, E^{1}\right)$ of formal symbols with coefficients in the bundle $\operatorname{Hom}\left(E^{0}, E^{1}\right)$ by local expressions in local coordinates on $X$ and local frames of $E^{0}, E^{1}$ and transition rules under change of coordinates and frames. A local expression for a formal symbol $\bar{a} \in \mathcal{A}\left(X, E^{0}, E^{1}\right)$ is a formal power series

$$
\bar{a}=\bar{a}(q, p, h)=\sum_{k=0}^{\infty} h^{k} a_{k}(q, p)
$$

in powers of a formal parameter $h$, whose coefficients $a_{k}(q, p)$ are smooth $\left(m^{1} \times m^{0}\right)$-matrix-valued functions in $(q, p) \in U \times \mathbb{R}^{n}$ where $U \subset X$ is a coordinate neighborhood, $q=\left(q^{1}, q^{2} \ldots, q^{n}\right) \in U$ and $p=\left(p_{1}, p_{2}, \ldots, p_{n}\right) \in$ $\mathbb{R}^{n}$. The term $a_{0}(q, p)$ is called the leading symbol of $\bar{a}$. For $\bar{a} \in \mathcal{A}\left(X, E^{1}, E^{2}\right)$ and $\bar{b} \in \mathcal{A}\left(X, E^{0}, E^{1}\right)$ the Leibniz product is defined by

$$
\bar{a} \# \bar{b}=\sum_{|\alpha|, k, l=0}^{\infty} h^{|\alpha|+k+l} \frac{(-i)^{|\alpha|}}{\alpha !} \frac{\partial^{\alpha} a_{k}}{\partial p^{\alpha}} \frac{\partial^{\alpha} b_{l}}{\partial q^{\alpha}} .
$$

This is an associative product (provided the ranks of vector bundles are compatible).

Let $U^{\prime}$ be another coordinate neighborhood and $q^{\prime}=f(q)$ in $U^{\prime} \cap U$. Set

$$
p^{\prime}=p\left(\frac{\partial f}{\partial q}\right)^{-1}
$$

where $p=\left(p_{1}, p_{2}, \ldots, p_{n}\right)$ means a row and $\partial f / \partial q$ means the differential (Jacobi matrix) of $f$. The transformation law

$$
\varphi:(q, p) \mapsto\left(q^{\prime}, p^{\prime}\right)=\left(f(q), p\left(\frac{\partial f}{\partial q}\right)^{-1}\right)
$$

means that the pairs $(q, p)$ may be considered as local coordinates on the manifold $M=T^{*} X$, the total space of the cotangent bundle of $X$. 
The transition rule for formal symbols under the transformation (1.3) is as follows. We introduce first a formal differential operator $\mathcal{L}$ on the space of formal symbols by

$$
\mathcal{L} \bar{a}=\left.\sum_{|\alpha|=0}^{\infty} \frac{(-i h)^{|\alpha|}}{\alpha !} \frac{\partial^{\alpha} \bar{a}}{\partial p^{\alpha}} \frac{\partial^{\alpha}}{\partial t^{\alpha}} \exp \frac{i}{h}\langle p, r(q, t)\rangle\right|_{t=0}
$$

where

$$
r(q, t)=\left(\frac{\partial f(q)}{\partial q}\right)^{-1}\left(f(q+t)-f(q)-\frac{\partial f(q)}{\partial q} t\right)
$$

and

$$
\langle p, r\rangle=p_{k} r^{k}(q, t)
$$

The formula for $\mathcal{L}$ may be rewritten as

$$
\mathcal{L} \bar{a}=\sum_{|\alpha|=0}^{\infty} \phi_{\alpha}(q, p, h) \frac{\partial^{\alpha}}{\partial p^{\alpha}} \bar{a}(q, p, h)
$$

with

$$
\phi_{\alpha}=\left.\frac{(-i h)^{|\alpha|}}{\alpha !} \frac{\partial^{\alpha}}{\partial t^{\alpha}} \exp \frac{i}{h}\langle p, r(q, t)\rangle\right|_{t=0} .
$$

Since $r(q, t)$ has a zero at $t=0$, the coefficients $\phi_{\alpha}$ are polynomials in $h$. Moreover, $t=0$ is a second-order zero of $r(q, t)$ implying that all monomials in $\phi_{\alpha}$ have degree at least $|\alpha| / 2$ in $h$. Thus, the series (1.5) makes sense as a formal series in $h$. We have also $\phi_{0} \equiv 1$ and $\phi_{\alpha}=0$ if $|\alpha|=1$. Thus,

$$
\mathcal{L} \bar{a}=\bar{a}+\sum_{|\alpha| \geq 2} \phi_{\alpha} \frac{\partial^{\alpha} \bar{a}}{\partial p^{\alpha}} .
$$

Now the transformation rule for formal symbols under the change of variables (1.3) is defined by

$$
\bar{a}\left(q^{\prime}, p^{\prime}, h\right)=\left(\varphi^{-1}\right)^{*}(\mathcal{L} \bar{a}(q, p, h))=\left.\mathcal{L} \bar{a}(q, p, h)\right|_{(q, p)=\varphi^{-1}\left(q^{\prime}, p^{\prime}\right)} .
$$

The right-hand side of (1.7) will be denoted $S_{\varphi} \bar{a}$ for short. In particular, the leading symbol $a_{0}$ changes as a function on $M=T^{*} X$, while the higher order coefficients change in a more complicated manner. 
Finally, for local isomorphisms

$$
V_{i}: E^{i} \rightarrow E^{i}, \quad i=0,1
$$

of vector bundles we define the corresponding transformation rule for symbols

$$
\bar{a} \mapsto V_{1}(q) \# \bar{a}(q, p, h) \# V_{0}^{-1}(q) .
$$

The following proposition describes basic properties of the transformation $(1.7)$.

Proposition 1.1 $S_{\varphi}$ is an automorphism of the algebra $\mathcal{A}(X)$, that is

$$
S_{\varphi}(\bar{a} \# \bar{b})=S_{\varphi}(\bar{a}) \# S_{\varphi}(\bar{b}) .
$$

Moreover, for two transformations $\varphi_{1}, \varphi_{2}$ of the type (1.3)

$$
S_{\varphi_{2}}\left(S_{\varphi_{1}} \bar{a}\right)=S_{\varphi_{2} \varphi_{1}}(\bar{a}) .
$$

We will prove this proposition a little bit later (see also [5]). Clearly, the same properties hold for (1.8).

We next introduce a trace ideal $\mathcal{J} \subset \mathcal{A}(X, E, E)$ and define a trace functional

$$
\left.\operatorname{Tr}: \mathcal{J} \rightarrow \mathbb{C}\left[h^{-1}, h\right]\right]
$$

where the notation on the right means formal Laurent series in $h$ with finite number of negative exponents.

Definition 1.2 Let $X$ be a compact manifold and $E^{0}=E^{1}=E . \quad A$ formal symbol $\bar{a} \in \mathcal{A}(X, E, E)$ belongs to a trace ideal $\mathcal{J}$ if its coefficients $a_{k}(q, p, h)$ vanish for $|p|$ large enough (this property is invariant with respect to transformations (1.7), (1.8)).

For $\bar{a} \in \mathcal{J}$ define

$$
\operatorname{Tr} \bar{a}=\frac{1}{(2 \pi h)^{n}} \int_{X}\left(\int_{\mathbb{R}^{n}} \operatorname{tr} \bar{a}(q, p, h) d p\right) d q
$$

where tr means the trace of matrix-valued coefficients. It follows from (1.6), (1.7) that after the inner integration over $\mathbb{R}^{n}$ we obtain a density on $X$ (independently of the choice of local coordinates), so that $\operatorname{Tr} \bar{a}$ is correctly defined. One easily checks that $\operatorname{Tr} \bar{a} \# \bar{b}=\operatorname{Tr} \bar{b} \# \bar{a}$. 
Definition 1.3 A formal symbol $\bar{a} \in \mathcal{A}\left(X, E^{0}, E^{1}\right)$ is called elliptic if there exists a symbol $\bar{r} \in \mathcal{A}\left(X, E^{1}, E^{0}\right)$ such that

$$
1-\bar{r} \# \bar{a} \in \mathcal{J} \subset \mathcal{A}\left(X, E^{0}, E^{0}\right)
$$

and

$$
1-\bar{a} \# \bar{r} \in \mathcal{J} \subset \mathcal{A}\left(X, E^{1}, E^{1}\right) .
$$

For elliptic elements an algebraic index is defined by

$$
\text { ind } \bar{a}=\operatorname{Tr}(1-\bar{r} \# \bar{a})-\operatorname{Tr}(1-\bar{a} \# \bar{r}) \text {. }
$$

The main property of the algebraic index is its stability: if $\bar{a}(t)$ is a smooth family of elliptic formal symbols, then ind $\bar{a}(t)$ is independent of $t$. As a consequence we come to the following proposition [5].

Proposition 1.4 The formal Laurent series (1.11) consists of the constant term only, that is ind $\bar{a}$ may be treated as a number.

We use formal symbols to approximate pseudo-differential operators on $X$. Let $\bar{a} \in \mathcal{A}\left(X, E^{0}, E^{1}\right)$ be a formal symbol satisfying the following additional assumption: the coefficients $a_{k}(q, p)$ are homogeneous functions of degree $m-k$ in $p$ for $|p|$ large enough, that is

$$
a_{k}(q, t p)=t^{m-k} a_{k}(q, p), m \in \mathbb{R},
$$

for $t \geq 1$ and $|p|$ large enough. Clearly, this property is invariant with respect to (1.7), the number $m$ being called the order. Let further $A$ be a classical $\psi$ do of order $m$ on $X$. We say that the operator $A$ and the formal symbol $\bar{a}$ are compatible if the asymptotic series

$$
a(q, p) \sim \sum_{k=0}^{\infty} a_{k}(q, p):=\bar{a}(q, p, 1)
$$

defines a complete symbol of the operator $A$ in local coordinates. This definition clarifies the meaning of the formal symbol. At large $|p|$ the formal symbol is almost the same as the complete symbol of a pseudo-differential operator $A$. But for small $|p|$ the notion of complete symbol becomes meaningless while the formal symbol is still meaningful. The powers of $h$ serve 
to order the terms of a formal symbol since the ordering by the degree of homogeneity is meaningless for small $|p|$. Loosely speaking, the formal symbol compatible with a $\psi$ do $A$ is its complete symbol defined for the whole $\mathbb{R}_{p}^{n}$. This correspondence does not depend on the choice of local coordinates because the rule (1.7) considered as an asymptotic formula at $h=1$ and $|p| \rightarrow \infty$ gives precisely the change of variables for complete symbols.

Given a classical $\psi$ do $A$ of order $m$ and a formal symbol $\bar{a}$ compatible with $A$, we construct an approximation $\mathrm{Op}\left(\left.\bar{a}\right|_{N}\right)$ as follows. Chose a coordinate covering $\left\{U_{i}\right\}$ of $X$ and a partition of unity $\left\{\rho_{i}\right\}$ subordinate to this covering. Let $\widetilde{\rho}_{i}$ be functions such that $\widetilde{\rho}_{i} \rho_{i} \equiv \rho_{i}$ and still supp $\widetilde{\rho}_{i} \subset U_{i}$. Then we set

$$
\operatorname{Op}\left(\left.\bar{a}\right|_{N}\right)=\sum_{i} \rho_{i} \mathrm{Op}\left(\sum_{k=0}^{N-1} a_{k}(q, p)\right) \widetilde{\rho}_{i}
$$

where Op on the right-hand side means a standard $\psi$ do on $\mathbb{R}^{n}$

$$
\mathrm{Op}(b(q, p)) u(q)=\frac{1}{(2 \pi)^{n}} \int_{\mathbb{R}^{n}} e^{i\langle p, q\rangle} a(q, p) \widehat{u}(p) d p .
$$

The compatibility condition means that the operator $A-\mathrm{Op}\left(\left.\bar{a}\right|_{N}\right)$ has the order $m-N$ for any $N$.

The correspondence between $\psi$ do and formal symbols becomes especially simple for the case of differential operators

$$
A=\sum_{|\alpha| \leq m} a_{\alpha}(q)\left(-i \frac{\partial}{\partial q}\right)^{\alpha}
$$

The formal symbol compatible with $A$ is

$$
h^{m} \exp \left(-\frac{i}{h}\langle p, q\rangle\right) A \exp \frac{i}{h}\langle p, q\rangle=\sum_{|\alpha| \leq m} h^{m-|\alpha|} a_{\alpha}(q) p^{\alpha} .
$$

The approximation (1.13) for $N>m$ does not depend on the choice of $\left\{U_{i}\right\},\left\{\rho_{i}\right\},\left\{\widetilde{\rho}_{i}\right\}$ and simply coincides with $A$. So, for differential operators we have a one-to-one correspondence between formal symbols (polynomials in $p$ ) and operators. As a consequence we obtain a simple proof of the Proposition 1.1. Indeed, it is sufficient to check relations (1.10) for formal symbols which are polynomials in $p$, that is for differential operators. But for the latter the 
transformation $S_{\varphi}$ is simply the change of variables, so the properties (1.9), (1.10) become evident. Being fulfilled for polynomials in $p$ they are valid for arbitrary formal symbols.

Returning to the general case and assuming $X$ compact, define a regularized trace of a $\psi$ do $A$ by

$$
\operatorname{Tr}_{N} A:=\operatorname{Tr}\left(A-\mathrm{Op}\left(\left.\bar{a}\right|_{N}\right)\right)
$$

where $N>m+n$, so that the operator on the right is of trace class.

Proposition 1.5 The regularized trace $\operatorname{Tr}_{N} A(N>m+n)$ has the following properties:

1. it is correctly defined, that is it depends only on $N$ but not on the choice of local coordinates, partition of unity $\left\{\rho_{i}\right\}$ and the functions $\left\{\widetilde{\rho}_{i}\right\}$;

2. it vanishes on commutators:

$$
\operatorname{Tr}_{N} A B=\operatorname{Tr}_{N} B A,
$$

provided the formal symbols $\bar{a} \# \bar{b}$ and $\bar{b} \# \bar{a}$ are used for approximations.

We finish this section with an algebraic index theorem for elliptic operators.

Theorem 1.6 Let $A$ be a classical elliptic operator on a compact manifold $X, \bar{a}$ a formal symbol compatible with $A$. Then the analytical index ind $A$ coincides with the algebraic index ind $\bar{a}$.

Proof. Let $R$ be a parametrix of $A$ up to trace class operators and $\bar{r}$ a formal symbol compatible with $R$. Then for $N$ large enough

$$
\begin{aligned}
& \text { ind } A=\operatorname{Tr}(1-R A)-\operatorname{Tr}(1-A R) \\
& =\operatorname{Tr} \operatorname{Op}\left(\left.(1-\bar{r} \# \bar{a})\right|_{N}\right)-\operatorname{Tr} \operatorname{Op}\left(\left.(1-\bar{a} \# \bar{r})\right|_{N}\right) \\
& -\operatorname{Tr}_{N} R A+\operatorname{Tr}_{N} A R .
\end{aligned}
$$

The last two terms here vanish because of (1.15) while the first two terms are equal to

$$
\operatorname{Tr}(1-\bar{r} \# \bar{a})-\operatorname{Tr}(1-\bar{a} \# \bar{r}):=\operatorname{ind} \bar{a} .
$$

We again refer to [5] for detailed proof of Proposition 1.5 and Theorem 1.6 . 


\section{Formal Symbols and Canonical Deforma- tion Quantization}

In this section we show that the algebra of formal symbols on the manifold $X$ may be obtained by a canonical construction of deformation quantization applied to a symplectic manifold $M=T^{*} X$. To this end we first introduce a coordinate-free description of the algebra $\mathcal{A}(X, E, E)$.

Let $\partial$ be a connection on $X$ and $\partial^{E}$ a connection on the bundle $E$. For any point $q \in X$ we introduce normal coordinates $u=\left(u^{1}, \ldots, u^{n}\right)$ in a neighborhood of $q$ and a normal frame of $E$ using connections $\partial$ and $\partial^{E}$. Recall that the normal coordinates are defined via the exponential map

$$
q^{\prime}=\exp _{q} u:=e_{q}(u)
$$

which assigns to a tangent vector $u \in T_{q} X$ the end point $q^{\prime}$ of the geodesics with the initial point $q$ and initial velocity $u$. The normal frame is obtained from a fixed frame in $E_{q}$ by parallel transports with respect to $\partial^{E}$ along geodesics. The normal coordinates are defined up to linear changes of variables corresponding to different choices of a basis in $T_{q} X$. Similarly, the normal frame is defined up to a choice of a basis in $E_{q}$.

Given a formal symbol $\bar{a} \in \mathcal{A}(X, E, E)$, we write it in normal coordinates obtaining a function $\bar{a}(q, u, p, h)$. Here we treat $(u, p) \in T_{q} X \oplus T_{q}^{*} X$ as symbol variables while $q \in X$ is considered as a parameter. Observe that all the coefficients $a_{k}(q, u, p)$ are correctly defined functions on $T_{q} X \oplus T_{q}^{*} X$ (for a fixed $q \in X)$ since for linear changes of normal coordinates the operator $\mathcal{L}$ in (1.7) reduces to identity. In a more geometric language, we introduce a bundle $L$ called the Leibniz bundle whose fiber at a point $q \in X$ is the algebra of formal symbols $\mathcal{A}\left(T_{q} X, E, E\right)$ on the tangent space (in normal coordinates). Then our family $\bar{a}(q, u, p, h)$ defines a section of this bundle. Strictly speaking, $u$ must be sufficiently small for exponential map to be defined. Another possibility is to treat $\bar{a}(q, u, p, h)$ as a formal Taylor series in $u$ :

$$
\bar{a}(q, u, p, h)=\sum_{|\alpha|=0}^{\infty} \bar{a}^{(\alpha)}(q, 0, p, h) u^{\alpha} / \alpha ! .
$$

A natural question arises: given a section $\bar{a}(q, u, p, h)$ of the bundle $L$, how should onefind out whether it comes from a global formal symbol $\bar{a} \in$ 
$\mathcal{A}(X, E, E)$ written in normal coordinates. The answer is that such a section is flat with respect to a special connection $\nabla$ on the bundle $L$. In what follows we denote by $d$ the de Rham differential with respect to $q \in X$, thus

$$
\begin{gathered}
d \bar{a}(q, u, p, h)=\frac{\partial \bar{a}}{\partial q^{i}} d q^{i} \\
d e_{q}(u)=\frac{\partial e_{q}(u)}{\partial q^{i}} d q^{i}
\end{gathered}
$$

and so on.

Theorem 2.1 If a section

$$
\bar{a}=\bar{a}(q)=\bar{a}(q, u, p, h) \in C^{\infty}(X, L)
$$

is generated by a global formal symbol $\bar{a} \in \mathcal{A}(X)$ it satisfies the equation

$$
\nabla \bar{a}:=d \bar{a}-\frac{i}{h}\left[\left\langle p,\left(\frac{\partial e_{q}(u)}{\partial u}\right)^{-1} d e_{q}(u)\right\rangle, a\right]=0
$$

where $[\cdot, \cdot]$ is a fiber-wise commutator in the algebra $L_{q}=\mathcal{A}\left(T_{q} X\right)$.

Proof. Introduce a fixed local coordinate system $q^{\prime}$ on $X$ and consider the change of variables (2.1) from normal coordinates $u$ at a point $q$ to $q^{\prime}$. We have a map (1.3)

$$
\left(q^{\prime}, p^{\prime}\right)=\varphi_{q}(u, p)
$$

or in more detail

$$
q^{\prime}=e_{q}(u), \quad p^{\prime}=p\left(\frac{\partial e_{q}(u)}{\partial u}\right)^{-1}
$$

where $q$ enters as a parameter. The symbol $\bar{a}\left(q^{\prime}, p^{\prime}, h\right) \in \mathcal{A}(X)$ may be rewritten by the transformation rule in the form

$$
\bar{a}\left(q^{\prime}, p^{\prime}, h\right)=\left.\mathcal{L}_{q} \bar{a}(q, u, p, h)\right|_{(u, p)=\varphi_{q}^{-1}\left(q^{\prime}, p^{\prime}\right)}:=S_{\varphi} \bar{a} .
$$

The differential of the right-hand side (with respect to $q$ ) must vanish since the left-hand side is independent of $q$. Thus, our necessary condition has the form

$$
S_{\varphi}^{-1} d\left(S_{\varphi} \bar{a}\right)=d \bar{a}+\left(S_{\varphi}^{-1} d S_{\varphi}\right) \bar{a}=0 .
$$


We now investigate the operator $S_{\varphi}^{-1} d S_{\varphi}$ on the Leibniz algebra $L_{q}$. First of all observe that $S_{\varphi}^{-1} d S_{\varphi}$ is a derivation since $S_{\varphi}$ is an automorphism of the algebra $L_{q}$ (Proposition 1.1). So it is sufficient to know its action on the generators of $L_{q}$, that is on functions $\bar{a}=b(u)$ and $\bar{a}=p_{i}$. The operator $\mathcal{L}$ given by (1.6) is an identity on these functions since higher-order derivatives in (1.6) vanish. So, $S_{\varphi}$ reduces to a pull-back operator $\left(\varphi^{-1}\right)^{*}$ with

$$
\varphi:(u, p) \mapsto\left(e_{q}(u), p\left(\frac{\partial e_{q}(u)}{\partial u}\right)^{-1}\right) .
$$

Now

$$
\begin{aligned}
& \left(S_{\varphi}^{-1} d S_{\varphi}\right) b(u)=\left.d b\left(e_{q}^{-1}\left(q^{\prime}\right)\right)\right|_{q^{\prime}=e_{q}(u)}=\left.\frac{\partial b(u)}{\partial u}\left(d e_{q}^{-1}\left(q^{\prime}\right)\right)\right|_{q^{\prime}=e_{q}(u)} \\
& =-\frac{\partial b(u)}{\partial u}\left(\frac{\partial e_{q}(u)}{\partial u}\right)^{-1} d e_{q}(u)=-\left\{\left\langle p,\left(\frac{\partial e_{q}(u)}{\partial u}\right)^{-1} d e_{q}(u)\right\rangle, b(u)\right\} \\
& =-\frac{i}{h}\left[\left\langle p,\left(\frac{\partial e_{q}(u)}{\partial u}\right)^{-1} d e_{q}(u)\right\rangle, b(u)\right] .
\end{aligned}
$$

Here $\{$,$\} means the Poisson bracket which for functions linear in p$ coincides with the commutator times $i / h$. Further,

$$
S_{\varphi} p_{i}=\left.\left\langle p^{\prime}, \frac{\partial e_{q}(u)}{\partial u^{i}}\right\rangle\right|_{u=e_{q}^{-1}\left(q^{\prime}\right)},
$$

so that

$$
\begin{aligned}
& S_{\varphi}^{-1} d\left(S_{\varphi} p_{i}\right)=\left\langle p,\left(\frac{\partial e_{q}(u)}{\partial u}\right)^{-1} d \frac{\partial e_{q}(u)}{\partial u^{i}}\right\rangle \\
& -\left\langle p,\left(\frac{\partial e_{q}(u)}{\partial u}\right)^{-1} \frac{\partial}{\partial u}\left(\frac{\partial e_{q}(u)}{\partial u_{i}}\right)\left(\frac{\partial e_{q}}{\partial u}\right)^{-1} d e_{q}(u)\right\rangle \\
& =\frac{\partial}{\partial u_{i}}\left\langle p,\left(\frac{\partial e_{q}(u)}{\partial u}\right)^{-1} d e_{q}(u)\right\rangle \\
& =-\left\{\left\langle p,\left(\frac{\partial e_{q}(u)}{\partial u}\right)^{-1} d e_{q}(u)\right\rangle, p_{i}\right\}
\end{aligned}
$$




$$
=-\frac{i}{h}\left[\left\langle p,\left(\frac{\partial e_{q}(u)}{\partial u}\right)^{-1} d e_{q}(u)\right\rangle, p_{i}\right],
$$

proving the theorem.

Before going further we make some remarks about the invariance of the operator

$$
\nabla \bar{a}=d \bar{a}-\frac{i}{h}\left[\left\langle p,\left(\frac{\partial e}{\partial u}\right)^{-1} d e\right\rangle, \bar{a}\right] .
$$

Formally, it depends on the choice of local coordinates $q^{\prime}$ on $X$ and the choice of frames in the tangent bundle $T X$. In fact, it does not depend on these choices, thus defining correctly a connection on the Leibniz bundle $L$.

Consider first a change of coordinates $q^{\prime \prime}=f\left(q^{\prime}\right)$. Then $(\partial e / \partial u)^{-1} d e$ should be replaced by

$$
\left(\frac{\partial f(e)}{\partial u}\right)^{-1} d f(e)=\left(\frac{\partial f}{\partial e} \frac{\partial e}{\partial u}\right)^{-1} \frac{\partial f}{\partial e} d e
$$

so it does not vary.

Now consider a linear change of variables $u \mapsto f(q) u$ in tangent spaces $T_{q} X$ where $f(q)$ is a non-degenerate matrix depending on $q \in X$. That is we replace the map $q^{\prime}=e_{q}(u)$ by $q^{\prime}=e_{q}(f u)$. Denoting

$$
e_{q}^{\prime}(u)=\frac{\partial e_{q}(u)}{\partial u}
$$

we would have

$$
\frac{\partial e_{q}(f u)}{\partial u}=e_{q}^{\prime}(f u) f
$$

and

$$
d\left(e_{q}(f u)\right)=\left(d e_{q}\right)(f u)+e_{q}^{\prime}(f u) d f u .
$$

Thus, $\left\langle p,(\partial e / \partial u)^{-1} d e\right\rangle$ should be replaced by

$$
\left\langle p f^{-1},\left(\left(e^{\prime}\right)^{-1} d e\right)(f u)\right\rangle+\left\langle p, f^{-1} d f u\right\rangle .
$$


On the other hand,

$$
\begin{aligned}
& d \bar{a}\left(q, f u, p f^{-1}, h\right)=(d \bar{a})\left(q, f u, p f^{-1}, h\right) \\
& +\frac{\partial \bar{a}}{\partial u}\left(q, f u, p f^{-1}, h\right) d f u-p f^{-1} d f f^{-1} \frac{\partial a}{\partial p}\left(q, f u, p f^{-1}, h\right) \\
& =(d \bar{a})\left(q, f u, p f^{-1}, h\right) \\
& +\frac{i}{h}\left[\left\langle p, f^{-1} d f u\right\rangle, \bar{a}\left(q, f u, p f^{-1}, h\right)\right] .
\end{aligned}
$$

The non-invariant second summand here cancels against the non-invariant second summand in (2.7), so that $\nabla \bar{a}$ remains invariant.

Proposition 2.2 The connection $\nabla$ is flat, that is

$$
\nabla^{2} \equiv 0
$$

Proof. We have using (2.5)

$$
\nabla^{2} a=S_{\varphi}^{-1} d\left(d\left(S_{\varphi} a\right)\right)=0 .
$$

Remark 2.3 We will need a stronger property of the connection $\nabla$. Denoting the connection one-form of the connection $\nabla$ by

$$
\theta=-\frac{i}{h}\left\langle p,\left(\frac{\partial e}{\partial u}\right)^{-1} d e\right\rangle
$$

we have

$$
d \theta+\theta^{2}=0 .
$$

Note, that (2.8) means that the curvature (2.9) belongs to the center but not necessarily vanishes.

To prove (2.9), consider

$$
d \theta=-\frac{i}{h}\left\langle p, d\left(\frac{\partial e}{\partial u}\right)^{-1} \wedge d e\right\rangle
$$


and

$$
\begin{aligned}
& \theta^{2}=\theta \# \theta=\frac{i}{h} \frac{\partial \theta}{\partial p_{i}} \wedge \frac{\partial \theta}{\partial u^{i}} \\
& =-\frac{i}{h}\left\langle p, \frac{\partial}{\partial u^{i}}\left(\left(\frac{\partial e}{\partial u}\right)^{-1} d e\right) \wedge\left(\left(\frac{\partial e}{\partial u}\right)^{-1} d e\right)^{i}\right\rangle \\
& =-\frac{i}{h}\left\langle p,\left(\frac{\partial e}{\partial u}\right)^{-1} d\left(\frac{\partial e}{\partial u^{i}}\right) \wedge\left(\left(\frac{\partial e}{\partial u}\right)^{-1} d e\right)^{i}\right\rangle \\
& +\frac{i}{h}\left\langle p,\left(\frac{\partial e}{\partial u}\right)^{-1} \frac{\partial^{2} e}{\partial u^{i} \partial u^{j}}\left(\left(\frac{\partial e}{\partial u}\right)^{-1} d e\right)^{i} \wedge\left(\left(\frac{\partial e}{\partial u}\right)^{-1} d e\right)^{j}\right\rangle .
\end{aligned}
$$

The second term here is equal to 0 because of the symmetry in $i, j$, while the first one is

$$
\frac{i}{h}\left\langle p, d\left(\frac{\partial e}{\partial u}\right)^{-1} \wedge d e\right\rangle .
$$

Together with $d \theta$ this term gives 0 proving (2.9).

The next step is to show that flat sections of Leibniz bundle are in one-toone correspondence with formal symbols. Here we repeat the arguments of canonical deformation quantization with the Leibniz bundle over $X$ instead of the Weyl bundle over $M=T^{*} X$.

So, we consider the bundle $L$ over $X$ whose sections are "functions"

$$
\bar{a}(q, u, p, h)=\sum_{k,|\alpha|=0}^{\infty} h^{k} a_{k \alpha}(q, p) u^{\alpha}
$$

$\left(q \in X, p \in T_{q}^{*} X, u=\left(u^{1}, \ldots, u^{n}\right) \in T_{q} X\right)$ considered as formal power series in $h$ and $u$ with $\operatorname{deg} h=1, \operatorname{deg} u^{i}=1$. The coefficients $a_{k \alpha}(q, p)$ are smooth functions on $T^{*} X$ with values in covariant tensor fields on $X$. Fixing $q \in X$, we obtain a fiber of $L$ consisting of "functions" on $(p, u) \in T_{q}^{*} X \oplus T_{q} X:=\Sigma_{q}$. We have a fiber-wise Leibniz product on sections

$$
a \# b=\sum_{|\alpha|=0}^{\infty} \frac{(-i h)^{|\alpha|}}{\alpha !} \frac{\partial^{\alpha} a}{\partial p^{\alpha}} \frac{\partial^{\alpha} b}{\partial u^{\alpha}}
$$


and a connection $\nabla$ defined by (2.6). Using the exponential map

$$
e_{q}^{i}(u)=q^{i}+u^{i}-\frac{1}{2} \Gamma_{j k}^{i} u^{j} u^{k}+\ldots
$$

we obtain

$$
\left(\left(\frac{\partial e}{\partial u}\right)^{-1} d e\right)^{i}=d q^{i}+\Gamma_{j k}^{i} u^{j} d q^{k}+\ldots
$$

where dots mean the terms of degree greater than 1 . Thus, the connection $\nabla$ may be represented as

$$
\nabla a=-\delta+(\nabla+\delta)
$$

where

$$
\delta=\frac{i}{h}[\langle p, d q\rangle, a]=\frac{\partial a}{\partial u^{i}} d q^{i}
$$

lowers degrees by 1 while the remainder $\nabla+\delta$ do not lower the degrees.

We also consider differential forms on $X$ with values in $L$, that is sections of the bundle $L \otimes \Lambda$ where $\Lambda$ is the bundle of exterior forms on $T X$. Further, we introduce an operator

$$
\delta^{*}=u^{k} i\left(\frac{\partial}{\partial q^{k}}\right)
$$

on forms which raises degrees in $h, u$ by $1, i$ being a convolution operator. Denoting by $\bar{a}_{k l}$ the homogeneous part of the form $\bar{a} \in C^{\infty}(L \otimes \Lambda)$ having the degree $k$ in $u$ and the exterior degree $l$, we have the following lemma similar to [4, Lemma 5.1.2].

Lemma 2.4 The operators $\delta$ and $\delta^{*}$ have the following properties:

$$
\begin{gathered}
\delta^{2}=\left(\delta^{*}\right)^{2}=0, \\
\left(\delta \delta^{*}+\delta^{*} \delta\right) a_{k l}=(k+l) a_{k l} .
\end{gathered}
$$

Setting

$$
\begin{gathered}
\delta^{-1} a_{k l}=\frac{1}{k+l} \delta^{*} a_{k l} \quad k+l>0 \\
\delta^{-1} a_{00}=0,
\end{gathered}
$$


we come to a representation

$$
a=\delta \delta^{-1} a+\delta^{-1} \delta a+a_{00}
$$

similar to the Hodge-de Rham decomposition.

The following proposition is quite similar to [4, Theorem 5.2.4].

Proposition 2.5 For any function

$$
a_{0}(q, p, h)=\sum_{k=0}^{\infty} h^{k} a_{k}(q, p) \in C^{\infty}\left(T^{*} X\right)[[h]]
$$

there exists a unique section $\bar{a}(q, u, p, h)$ of the Leibniz bundle with

$$
\left.\bar{a}(q, u, p, h)\right|_{u=0}=a_{0}(q, p, h) .
$$

We refer the reader to [4, Theorem 5.2.4] for the proof.

Let us continue to study the correspondence between formal symbols and flat sections. Since the constructions are local, we restrict ourselves to a fixed local coordinate system $q^{\prime}=\left(q^{1^{\prime}}, q^{2^{\prime}}, \ldots, q^{n^{\prime}}\right)$ on $X$, so formal symbols are simply functions $\bar{a}\left(q^{\prime}, p^{\prime}, h\right)$. The change of variables to normal coordinates $q^{\prime}=e_{q}(u)$ gives us a symbol $\bar{a}(q, u, p, h)$ such that $\nabla \bar{a}(q, u, p, h) \equiv 0$. We have

$$
\bar{a}\left(q^{\prime}, p^{\prime}, h\right)=\left(\varphi^{-1}\right)^{*}\left(\mathcal{L}_{q} \bar{a}(q, u, p, h)\right)
$$

with $\varphi$ given by (2.3) and

$$
\mathcal{L}_{q} \bar{a}=\bar{a}+\sum_{|\alpha| \geq 2} \phi_{\alpha}(q, u, h) \frac{\partial^{\alpha} \bar{a}}{\partial p^{\alpha}}
$$

with $\operatorname{deg} \phi_{\alpha} \geq|\alpha| / 2$ in $h$. Setting $q=q^{\prime}, u=0$ in (2.11), we obtain

$$
\bar{a}\left(q^{\prime}, p^{\prime}, h\right)=\mathcal{L}_{q^{\prime}}\left(0, \frac{\partial}{\partial p^{\prime}}, h\right) \bar{a}\left(q^{\prime}, 0, p^{\prime}, h\right) .
$$

The operator $\mathcal{L}$ is invertible as a formal series in $h$, so

$$
\bar{a}\left(q^{\prime}, 0, p^{\prime}, h\right)=\mathcal{L}_{q^{\prime}}^{-1}\left(0, \frac{\partial}{\partial p^{\prime}}, h\right) \bar{a}\left(q^{\prime}, p^{\prime}, h\right) .
$$

We come to the following proposition. 
Proposition 2.6 For any flat section $\bar{a}(q, u, p, h)$ of the Leibniz bundle there exists a unique formal symbol $\bar{a}\left(q^{\prime}, p^{\prime}, h\right)$ such that its expression in the normal coordinates $q^{\prime}=e_{q}(u)$ coincides with the given flat section (as a formal Taylor expansion at $u=0$ ).

Proof. Given a flat section $\bar{a}(q, u, p, h)$, we define the symbol $\bar{a}\left(q^{\prime}, p^{\prime}, h\right)$ by (2.13). Writing this symbol in normal coordinates, we obtain another flat section, let us denote it by $\bar{a}_{1}(q, u, p, h)$. But from (2.14) it follows that

$$
\bar{a}(q, 0, p, h)=\bar{a}_{1}(q, 0, p, h),
$$

so by Proposition 2.5 both flat sections coincide.

The construction of Leibniz bundle and its flat sections is very similar to the canonical deformation quantization construction except two things:

1. in deformation quantization we deal with the Weyl algebra bundle $W$ instead of the Leibniz bundle $L$,

2. the bundle $W$ is defined over the phase manifold $M$, that is over $T^{*} X$, rather than over $X$.

But these two differences can be easily removed. We begin with item 2 . Replace the variable $p \in T_{q}^{*} X$ in $\bar{a}(q, u, p, h)$ by $p+v$, where $p, v \in T_{q}^{*} X$. We obtain a "function"

$$
\bar{b}(q, p ; u, v ; h)=\bar{a}(q, u, p+v, h) .
$$

The Leibniz product of $a_{1}(q, u, p, h)$ and $a_{2}(q, u, p, h)$ with respect to $u, p$ under this replacement corresponds to the Leibniz product of

$$
\bar{b}_{1}(q, p ; u, v ; h)=\bar{a}_{1}(q, u, p+v, h)
$$

and

$$
\bar{b}_{2}(q, p ; u, v ; h)=\bar{a}_{2}(q, u, p+v, h)
$$

with respect to variables $u, v$ :

$$
b_{1} \# b_{2}=\sum \frac{(-i h)^{|\alpha|}}{\alpha !} \frac{\partial^{\alpha} b_{1}}{\partial v^{\alpha}} \frac{\partial^{\alpha} b_{2}}{\partial u^{\alpha}},
$$


while $p \in T_{q}^{*} X$ becomes a new parameter. Thus, we obtain the Leibniz bundle over the phase space $(q, p) \in T^{*} X$. A special dependence on $p$ and $v$ (2.15) means that

$$
\frac{\partial \bar{b}}{\partial p_{i}} d p_{i}=\frac{\partial \bar{b}}{\partial v_{i}} d p_{i}=-\frac{i}{h}[\langle d p, u\rangle, \bar{b}]
$$

or

$$
\frac{\partial \bar{b}}{\partial p_{i}} d p_{i}+\frac{i}{h}[\langle d p, u\rangle, \bar{b}]=0 .
$$

So, formal symbols $\bar{a} \in \mathcal{A}(X, E, E)$ correspond now to sections $\bar{b}(q, p ; u, v ; h)$ of the Leibniz bundle $L$ lifted to $T^{*} X$, satisfying two conditions: the first one is (2.16) and the second is the modified condition $\nabla \bar{a}=0$, that is

$$
\frac{\partial \bar{b}}{\partial q^{i}} d q^{i}-\frac{i}{h}\left[\left\langle p+v,\left(\frac{\partial e}{\partial u}\right)^{-1} d e\right\rangle, \bar{b}\right]=0 .
$$

Denoting from now on by

$$
d=d q^{i} \wedge \frac{\partial}{\partial q^{i}}+d p_{i} \wedge \frac{\partial}{\partial p_{i}}
$$

the de Rham differential on the manifold $M=T^{*} X$, we can unify (2.16) and (2.17) as

$$
D \bar{b}:=d \bar{b}+\frac{i}{h}\left[\langle d p, u\rangle-\left\langle p+v,\left(\frac{\partial e}{\partial u}\right)^{-1} d e\right\rangle, \bar{b}\right]=0 .
$$

Thus, the formal symbols $\bar{a} \in \mathcal{A}(X)$ correspond to flat sections $\bar{b}(q, p ; u, v ; h)$ of the Leibniz bundle over $M=T^{*} X$ with respect to a connection $D$ which is obviously flat.

Now, we want to replace the Leibniz bundle by the Weyl bundle. It is quite easy since the Leibniz product

$$
a(u, v) \# b(u, v)=\left.\exp \left(-i h \frac{\partial}{\partial \tau} \frac{\partial}{\partial t}\right) a(u, v+\tau) b(u+t, v)\right|_{t=\tau=0}
$$

and the Weyl product

$$
\begin{aligned}
& a \circ b=\exp \left(-\frac{i h}{2}\left(\frac{\partial}{\partial \tau_{1}} \frac{\partial}{\partial t_{2}}-\frac{\partial}{\partial \tau_{2}} \frac{\partial}{\partial t_{1}}\right)\right) \\
& \left.a\left(u+t_{1}, v+\tau_{1}\right) b\left(u+t_{2}, v+\tau_{2}\right)\right|_{t_{1}=t_{2}=\tau_{1}=\tau_{2}=0}
\end{aligned}
$$

are equivalent. 


\section{Proposition 2.7 Let}

$$
I \bar{a}=\exp \left(\frac{i h}{2} \frac{\partial^{2}}{\partial v_{i} \partial u^{i}}\right) \bar{a}(u, v) .
$$

Then

$$
I a \circ I b=I(a \# b) .
$$

Proof. Consider a function

$$
\bar{a}\left(a+t_{1}, v+\tau_{1}\right) \bar{b}\left(u+t_{2}, v+\tau_{2}\right),
$$

we drop the variables $q, p, h$ since only fiber-wise relations are considered. To obtain the left-hand side of (2.20), we apply to (2.21) the operator

$$
\exp \left(\frac{i h}{2} \frac{\partial^{2}}{\partial \tau_{1} \partial t_{1}}\right) \exp \left(\frac{i h}{2} \frac{\partial^{2}}{\partial \tau_{2} \partial t_{2}}\right) \exp \left(-\frac{i h}{2}\left(\frac{\partial^{2}}{\partial \tau_{1} \partial t_{2}}-\frac{\partial^{2}}{\partial \tau_{2} \partial t_{1}}\right)\right)
$$

and then put $t_{1}=t_{2}=\tau_{1}=\tau_{2}=0$. To obtain the right-hand side apply the operator

$$
\exp \frac{i h}{2}\left(\frac{\partial^{2}}{\partial \tau_{1} \partial t_{1}}+\frac{\partial^{2}}{\partial \tau_{2} \partial t_{2}}+\frac{\partial^{2}}{\partial \tau_{1} \partial t_{2}}+\frac{\partial^{2}}{\partial \tau_{2} \partial t_{1}}\right) \exp \left(-i h \frac{\partial^{2}}{\partial \tau_{1} \partial t_{2}}\right)
$$

and put $t_{1}=t_{2}=\tau_{1}=\tau_{2}=0$. But these both operators coincide, proving the proposition.

Let us summarize our investigations. We have constructed a symplectic bundle $\Sigma$ over the manifold $M=T^{*} X$. The fiber of $\Sigma$ above the point $x=(q, p)$ where $q \in X, p \in T_{q}^{*} X$ is

$$
\Sigma_{x}=T_{q} X \oplus T_{q}^{*} X
$$

with a standard symplectic form. On the fibers of $\Sigma$ there is a Weyl algebra structure $W\left(\Sigma_{x}\right):=W_{x}$ with the product $\circ$, and the union of $W_{x}$ forms the Weyl algebra bundle $W$ over $M$. Further, we have a connection $D$ on $W$

$$
D \bar{a}=d \bar{a}+\frac{i}{h}[I \gamma, \bar{a}]=d \bar{a}+\frac{i}{h}(I \gamma \circ \bar{a}-\bar{a} \circ I \gamma)
$$


where

$$
I \gamma=\gamma+\frac{i h}{2} \frac{\partial^{2}}{\partial v_{i} \partial u^{i}} \gamma
$$

and

$$
\gamma=\langle d p, u\rangle-\left\langle p+v,\left(\frac{\partial e}{\partial u}\right)^{-1} d e\right\rangle .
$$

The connection $D$ is flat on $W$, so we are in the setting of the canonical deformation quantization on $M$. It remains to find the equivalence class of this deformation quantization. In other words, we need to calculate the Weyl curvature of the connection $D$.

Proposition 2.8 If $\partial$ is a Riemannian connection on $X$, then the Weyl curvature of $D$ is equal to

$$
\Omega=-\langle d p, d q\rangle .
$$

Proof. Recall the notion of the Weyl curvature [4]. First we need to normalize the connection form $I \gamma$ by subtracting its constant term

$$
(I \gamma)_{0}=\left.(I \gamma)\right|_{u=v=o}
$$

Then by definition

$$
\begin{aligned}
& \Omega=d\left(I \gamma-(I \gamma)_{0}\right)+\frac{i}{h}\left(I \gamma-I \gamma_{0}\right) \circ\left(I \gamma-I \gamma_{0}\right) \\
& =-d(I \gamma)_{0}+d I \gamma+\frac{i}{h} I \gamma \circ I \gamma \\
& =-d(I \gamma)_{0}+I\left(d \gamma+\frac{i}{h} \gamma \# \gamma\right) .
\end{aligned}
$$

But in virtue of Remark 2.3

$$
d \gamma+\frac{i}{h} \gamma \# \gamma=0
$$

So

$$
\Omega=-d(I \gamma)_{0} .
$$

We will need a few terms of the expansion

$$
e_{q}^{i}(u)=q^{i}+u^{i}-\frac{1}{2} \Gamma_{j k}^{i}(q) u^{i} u^{j}+\ldots
$$


up to cubic terms in $u$. Then

$$
\left(\frac{\partial e_{q}(u)}{\partial u}\right)^{-1} d e_{q}(u)=d q^{i}+\Gamma_{j k}^{i} u^{j} d q^{k}+\ldots
$$

up to quadratic terms in $u$. Thus,

$$
(I \gamma)_{0}=p_{i} d q^{i}+\frac{i h}{2} \Gamma_{i k}^{i} d q^{k}
$$

and

$$
\Omega=-d(I \gamma)_{0}=d p_{i} \wedge d q^{i}
$$

because

$$
\Gamma_{i k}^{i} d q^{k}=d \log \sqrt{|g|},
$$

so this term does not contribute to $\Omega$.

Thus, we have come to the canonical deformation quantization construction with a symplectic manifold $M=T^{*} X$, symplectic bundle $\Sigma=\pi^{*} T X \otimes$ $\pi^{*} T^{*} X$ and the symplectic connection on the Weyl algebra bundle $W=$ $W(\Sigma)$

$$
D=d+\frac{i}{h}\left[I \gamma-(I \gamma)_{0}, \cdot\right]
$$

with

$$
\gamma=\langle d p, u\rangle-\left\langle p+v,\left(\frac{\partial e}{\partial u}\right)^{-1} d e\right\rangle .
$$

The terms linear in $(u, v)$

$$
\gamma_{1}=\langle d p, u\rangle-\langle v, d q\rangle-\left\langle p_{i}, \Gamma_{j k}^{i} u^{j} d q^{k}\right\rangle
$$

define isomorphism between $\Sigma$ and the tangent bundle $T M$. The tangent vector $X \in T M$ goes to a vector $Y \in \Sigma$ such that

$$
\omega(Y, \cdot)=i(X) \gamma_{1}
$$

where $\omega$ is the symplectic form on $\Sigma$. The quadratic terms $\gamma_{2}$ of $\gamma$ define a symplectic connection on $\Sigma$ and thus a connection on $T M$ preserving the symplectic form $\langle d p, d q\rangle$. The Abelian property of $D$ implies

$$
d \gamma_{1}+\frac{i}{h}\left[\gamma_{2}, \gamma_{1}\right]=0
$$


which means that the connection on $T M$ is torsion-free. Of course, it is more convenient to deal with $W(\Sigma)$ than with the Weyl algebra bundle $W(T M)$ though everything may be transported from $W(\Sigma)$ to $W(T M)$ via isomorphism (2.23).

\section{Index Theorems}

In this section we first show that the isomorphism $Q: \mathcal{A}(X) \rightarrow W_{D}(M)$ between the algebra of formal symbols on $X$ and the algebra of flat sections on $T^{*} X$ constructed in the previous section preserves the traces. Again it is sufficient to restrict ourselves to a fixed coordinate neighborhood $O \subset X$ with coordinates $q^{\prime}=\left(q^{1^{\prime}}, q^{2^{\prime}}, \ldots, q^{n^{\prime}}\right)$. For a symbol $\bar{a}\left(q^{\prime}, p^{\prime}, h\right) \in \mathcal{A}(X)$ with support in $O$ we have

$$
\operatorname{Tr} \bar{a}=\frac{1}{(2 \pi h)^{n}} \int_{\mathbb{R}^{2 n}} \operatorname{tr} \bar{a}\left(q^{\prime}, p^{\prime}, h\right) d p^{\prime} d q^{\prime} .
$$

Consider now the isomorphism $Q$ constructed in Section 2 using a trivial connection on $X$, so that the exponential map has the form

$$
e_{q}^{i}(u)=q^{i}+u^{i}
$$

Then the flat section of the bundle $W(\Sigma)$ corresponding to the symbol $\bar{a}\left(q^{\prime}, p^{\prime}, h\right)$ is $\bar{a}(q+u, p+v, h)$. Its trace is given by (3.1) according to the definition of the trace [4, Section 5.6]. If we use a non-trivial connection to define an Abelian connection $D$ on the Weyl algebra bundle $W(\Sigma)$, then by the definition of the trace on $W_{D}[4$, Section 5.6] we need to trivialize the algebra $W_{D}$ locally to the standard Weyl algebra $W\left(\mathbb{R}^{2 n}\right)$. Such a trivialization may be achieved by taking a trivial connection on $X$ as was described above. The trace on $W_{D}$ is independent of the local trivializations, this gives the coincidence of traces in both algebras.

We could say here that the coincidence of traces implies the coincidence of indices, and thus finish our article, but for one fact which at the first glance seems rather technical and inessential. The matter is that there are two reasonable definitions of the index in deformation quantization. One of them comes from the general algebraic K-theory, another one is more specific for deformation quantization. In the rest of this paper we prove their equivalence. 
Consider first a more simple case of a compact manifold $M$ when the trace functional is defined on the whole algebra. Let $W_{D}(M) \cong C^{\infty}(M)[[h]]$ be a deformation of the algebra of scalar-valued functions $C^{\infty}(M)$. We will consider the matrices with entries in $W_{D}(M)$, that is the algebra $W_{D}(M) \otimes$ $\operatorname{Mat}(N)$. The order $N$ is not fixed, it may be enlarged using borderings by zeros. Let $\bar{P}$ be a projector in $W_{D}(M) \otimes$ Mat, that is $\bar{P} \circ \bar{P}=\bar{P}$ or

$$
\sum_{j=1}^{N} \bar{p}_{i j} \circ \bar{p}_{j k}=\bar{p}_{i k} .
$$

We associate to it an algebra $A_{\bar{P}}$ consisting of matrices $\bar{a}$ with entries from $W_{D}(M)$ and satisfying the following conditions

$$
\bar{a} \circ \bar{P}=\bar{P} \circ \bar{a}=\bar{a} .
$$

Together with (3.2) these conditions mean that $\bar{P} \in A_{\bar{P}}$ is the unit of the algebra $A_{\bar{P}}$. We set

$$
\text { ind } A_{\bar{P}}=\operatorname{Tr} \bar{P}=\sum_{i} \operatorname{Tr} \bar{p}_{i i} .
$$

Thus, the index is the trace of the unit element of the algebra. If the elements of the algebra were operators, the index would be equal to the dimension of the Hilbert space where the operators act, or, using physicist terminology, to the number of quantum states.

The stability of index implies that it depends only on the leading term of $\bar{P}$ which is a projector-valued function $P(x)$ on $M$. This function defines a vector bundle $E$ over $M$ whose fiber at a point $x$ is the range of the projector $P(x)$. So, a vector function $u(x)$ is a section of $E$ if it satisfies the relation

$$
u(x) \equiv P(x) u(x) .
$$

The projector $P(x)$ defines a canonical connection on $E$, the so-called LeviCivita connection. It acts on sections (3.5) as

$$
\partial^{E} u(x)=P(x) d u \text {. }
$$

Here $d$ is the de Rham differential, if $u(x)$ is represented as a column $\left(u_{1}(x), \ldots, u_{N}(x)\right)^{t}$, then $d u$ is a column with entries $d u_{k}(x)$. 
We can construct another algebra $A_{P}$ associated to the projector $P$ using the so-called quantization with a non-trivial coefficient bundle. Consider a bundle $K=\operatorname{Hom}(E, E)$ which we will refer to as a coefficient bundle. The section $a(x) \in C^{\infty}(M, K)$ may be represented as a matrix-valued function on $M$ such that

$$
P(x) a(x)=a(x) P(x)=a(x) .
$$

The connection (3.6) on $E$ gives a connection on $K$ denoted by the same symbol $\partial^{E}$ :

$$
\partial^{E} a=P d a P .
$$

The section $P(x)$ of the bundle $K$ is covariantly constant: $\partial^{E} P=P d P P \equiv 0$ and relations (3.7) mean that it is a unit section in $K$.

The curvature of the Levi-Civita connection (3.6) is $R^{E}=P d P d P$ and from (3.8) we obtain

$$
\left(\partial^{E}\right)^{2} a=\left[R^{E}, a\right] .
$$

Let us recall briefly the canonical construction of deformation quantization with a non-trivial coefficient bundle $K=\operatorname{Hom}(E, E)$. We consider the Weyl algebra bundle with values in $K$, that is the bundle $W \otimes K$. We have connections $\partial^{s}$, a symplectic connection acting on $W$, and $\partial^{E}$ acting on $K$. So, on $W \otimes K$ there is a connection

$$
\partial=\partial^{s} \otimes 1+1 \otimes \partial^{E}
$$

and

$$
\partial^{2} a=\frac{i}{h}\left[R^{s}-i h R^{E}, a\right] .
$$

Then we repeat the canonical construction taking for the curvature

$$
R=R^{s} \otimes 1-i h 1 \otimes R^{E} .
$$

Thus, we look for the Abelian connection $D_{P}$ on the bundle $W \otimes K$ in the form

$$
D_{P} a=-\delta a+\partial a+\frac{i}{h}\left[r_{P}, a\right]
$$

and come to the equation for $r_{P}$

$$
\delta r_{P}=R+\partial r_{P}+\frac{i}{h} r_{P}^{2}
$$


which may be solved by iterations:

$$
r_{P}=\delta^{-1} R+\delta^{-1}\left(\partial r_{P}+\frac{i}{h} r_{P}^{2}\right)
$$

(see [4, Section 5.2] for more detail). We denote this connection by $D_{P}$ reserving the notation $D$ for the Abelian connection on the bundle $W$ (that is for the trivial coefficient bundle). Define the algebra $A_{P}$ to be the subalgebra of flat sections:

$$
A_{P}=\left\{a \in C^{\infty}(M, W \otimes K): D_{P} a=0\right\} .
$$

A unit in the algebra $A_{P}$ is a unit section of $K$, that is $P(x)$. It may be easily verified that $D_{P} P(x) \equiv 0$ and for any $a \in A_{P}$ we have

$$
P a=a P=a .
$$

Similarly to the ind $A_{\bar{P}}$ we define the index of the algebra $A_{P}$ to be

$$
\text { ind } A_{P}=\operatorname{Tr} P(x) \text {, }
$$

that is, again the trace of a unit element, but the trace is understood in the algebra $A_{P}$.

Our aim in this section is to prove the equality

$$
\text { ind } A_{\bar{P}}=\text { ind } A_{P}
$$

which is a consequence of the following theorem.

Theorem 3.1 There exists an isomorphism betweem the algebras $A_{P}$ and $A_{\bar{P}}$ preserving the traces.

It is important that the isomorphism is defined globally on $M$ (locally such an isomorphism always exists).

Proof. Both algebras $A_{P}$ and $A_{\bar{P}}$ are subalgebras of $C^{\infty}(W(M) \otimes \mathrm{Mat})$, that is they both consist of matrix-valued sections of the bundle $W(M)$. In other words

$$
A_{P}=\left\{a \in C^{\infty}(W \otimes \mathrm{Mat}): P a=a P=a, D_{P} a \equiv 0\right\}
$$




$$
A_{\bar{P}}=\left\{\bar{a} \in C^{\infty}(W \otimes \mathrm{Mat}): D \bar{a} \equiv 0, \bar{P} \circ \bar{a}=\bar{a} \circ \bar{P}=\bar{a}\right\} .
$$

We construct the isomorphism in the form

$$
\bar{a}=U \circ a \circ V
$$

where $U, V$ are global sections of $W^{+} \otimes$ Mat satisfying the following conditions:

$$
\begin{gathered}
\bar{P} \circ U=U P=U \\
P V=V \circ \bar{P}=V \\
U \circ V=\bar{P} ; V \circ U=P .
\end{gathered}
$$

(Here $W^{+}$means the extension of $W$ consisting of Laurent power series in $h$ with positive total degrees of all terms, see [3]). Besides, for any $a \in A_{P}$ the element $\bar{a}$ must be flat with respect to $D$. This condition results in differential equations for $U$ and $V$. To derive them, apply $D$ to both sides of (3.14) obtaining

$$
0=(D U) \circ a \circ V+U \circ D a \circ V+U \circ a \circ D V .
$$

Because of $(3.15),(3.16)$ we may replace $D a$ by $P(D a) P, D U$ by $(D U) P$ and $D V$ by $P(D V)$. Denoting $\partial^{s}$ the symplectic connection and using

$$
P \partial^{s} a P=\partial a,
$$

we come to the following identity for $a \in A_{P}$

$$
P(D a) P=-\delta a+\partial a+\frac{i}{h}[r, a] .
$$

Here we have used the fact that $P$ is absorbed by $a \in A_{P}$ and commutes with $r$ since $r$ is a scalar-valued section of $W \otimes \Lambda^{1}$. Since $a$ is a flat section with respect to $D_{P},(3.19)$ results in

$$
P(D a) P=D_{P} a+\frac{i}{h}\left[r-r_{P}, a\right]=\frac{i}{h}\left[r-r_{P}, a\right]
$$

and (3.18) gives

$$
\begin{aligned}
& \left\{(D U) P+\frac{i}{h} U \circ\left(r-r_{P}\right)\right\} \circ a \circ V \\
& +U \circ a \circ\left\{P(D V)-\frac{i}{h}\left(r-r_{P}\right) \circ V\right\}=0 .
\end{aligned}
$$


We find $U$ and $V$ from the equations

$$
\begin{aligned}
& (D U) P+\frac{i}{h} U \circ\left(r-r_{P}\right)=0 \\
& P(\partial V)-\frac{i}{h}\left(r-r_{P}\right) \circ V=0 .
\end{aligned}
$$

Consider in more detail (3.20). There is a compatibility condition which is necessary for solvability of (3.20). Indeed, applying $D$ to the left-hand side and multiplying by $P$ from the right, we obtain

$$
\begin{aligned}
& (D((D U) P)) P+\frac{i}{h}(D U) P \circ\left(r-r_{P}\right) P \\
& +\frac{i}{h} U \circ\left(D\left(r-r_{P}\right)\right) P=0 .
\end{aligned}
$$

We use again that $P$ is absorbed in $U$ in virtue of (3.15). Further, since $D$ is an Abelian connection $D^{2} U=0$, so

$$
D((D U) P) P=-D U D P P=-U D P D P P,
$$

because $D U=D(U P)=(D U) P+U D P$ and the term $(D U) P$ vanishes since $D U P D P P=0$. Replacing $(D U) P$ by $-i / h U \circ\left(r-r_{P}\right) P$ from(3.20), we come to the identity

$$
U \circ\left\{\frac{i}{h} P D\left(r-r_{P}\right) P-D P D P P-\left(\frac{i}{h}\right)^{2} P\left(r-r_{P}\right)^{2} P\right\}=0 .
$$

This identity is fulfilled because of the equations for $r$ and $r_{P}$. Indeed,

$$
\begin{aligned}
& -\delta r_{P}+R^{s}-i h R^{E}+P \partial^{s} r_{P} P+\frac{i}{h} r_{P}^{2}=0 \\
& -\delta r+R^{s}+\partial^{s} r+\frac{i}{h} r^{2}=0,
\end{aligned}
$$

so taking the difference and multiplying by $P$ from both sides, we obtain

$$
-P \delta\left(r-r_{P}\right) P+\partial\left(r-r_{P}\right)+i h P d P d P+\frac{i}{h} P\left(r^{2}-r_{P}^{2}\right) P=0
$$


which is precisely the desired identity (3.22). To solve (3.20), we rewrite it in the form

$$
\delta U=((D+\delta) U) P+\frac{i}{h} U \circ\left(r-r_{P}\right) .
$$

Applying $\delta^{-1}$ and taking the leading term of $U$ to be equal to $P$, we come to the equation

$$
U=P+\delta^{-1}\left\{((D+\delta) U) P+\frac{i}{h} U \circ\left(r-r_{P}\right)\right\} .
$$

The only difficulty here is that $h$ enters the denominator but for the total degree we have

$$
\operatorname{deg}\left(\frac{i}{h}\left(r-r_{P}\right)\right) \geq 1 .
$$

So, the iteration procedure will be convergent with respect to total degrees. The solution of (3.23) also satisfies (3.20) because of the compatibility condition (cf. [3, Theorem 4.3]). All the iterations are not changed being multiplied by $P$ from the right. As for multiplication by $\bar{P}$ from the left, we see from (3.20) that for a solution $U$ the section $\bar{P} \circ U$ is again a solution which satisfies all the conditions (3.15).

To find $V$, one can solve (3.21) but it is easier to invert $U$. We take $V_{0}=P \circ \bar{P}$ as an initial approximation and then define

$$
V=\sum_{k=0}^{\infty}\left(P-V_{0} \circ U\right)^{k} \circ V_{0}=V_{0} \circ \sum_{k=0}^{\infty}\left(\bar{P}-U \circ V_{0}\right)^{k} .
$$

The pair $U, V$ satisfies (3.15)-(3.17) and gives the desired isomorphism of $A_{\bar{P}}$ and $A_{P}$.

To see that this isomorphism preserves the traces, consider a section $a \in$ $A_{P}$ with the support in a Darboux coordinate neighborhood $O$. To calculate the trace, we need to take a local isomorphism

$$
I_{P}:\left.A_{P}\right|_{0} \cong W\left(\mathbb{R}^{2 r}\right) \otimes \text { Mat }
$$

and then

$$
\operatorname{Tr} a_{P}=\frac{1}{(2 \pi h)^{n}} \int_{\mathbb{R}^{2 n}} \operatorname{tr} I_{P} a_{P} \frac{\omega^{n}}{n !} .
$$


Consider the image

$$
a_{\bar{P}}=U \circ a_{P} \circ V \in A_{\bar{P}}
$$

Similarly,

$$
\operatorname{Tr} a_{\bar{P}}=\frac{1}{(2 \pi h)^{n}} \int_{\mathbb{R}^{2} n} \operatorname{tr} I_{\bar{P}} a_{\bar{P}} \frac{\omega^{n}}{n !}
$$

where $I_{\bar{P}}$ is a local isomorphism of $A_{\bar{P}}$ to $W\left(\mathbb{R}^{2 n}\right) \otimes$ Mat. But the local isomorphisms $I_{P}$ and $I_{\bar{P}}$ may be obtained from each other by the global isomorphism

$$
a_{\bar{P}}=U \circ a_{P} \circ V
$$

so that

$$
I_{\bar{P}} a_{\bar{P}}=I_{\bar{P}}\left(U \circ a_{P} \circ V\right):=I_{P} a_{P}
$$

and vice versa, proving the coincidence of the traces.

Consider now the case when $M$ is not compact. In this case the Ktheoretical definition of the index looks as follows. The index is defined for pairs $\left\{\bar{P}_{0}, \bar{P}_{1}\right\}$ of projectors in the algebra $W_{D}(M) \otimes$ Mat for which the difference $\bar{P}_{0}-\bar{P}_{1}$ has compact support

$$
\text { ind }\left\{\bar{P}_{0}, \bar{P}_{1}\right\}=\operatorname{Tr}\left(\bar{P}_{0}-\bar{P}_{1}\right) \text {. }
$$

In particular, we come to this definition considering Fredholm operators

$$
A: C^{\infty}\left(X, E^{0}\right) \rightarrow C^{\infty}\left(X, E^{1}\right)
$$

and their parametrices

$$
R: C^{\infty}\left(X, E^{1}\right) \rightarrow C^{\infty}\left(X, E^{0}\right) .
$$

They define a pair of projectors

$$
\mathcal{P}_{0}=\left(\begin{array}{cc}
1-R A & R \\
A(1-R A) & A R
\end{array}\right) ; \mathcal{P}_{1}=\left(\begin{array}{cc}
0 & R \\
0 & 1
\end{array}\right)
$$

and ind $A=\operatorname{Tr}\left(\mathcal{P}_{0}-\mathcal{P}_{1}\right)$. Passing to formal symbols and then to deformation quantization, we come to the definition (3.24).

Another reasonable definition of the index in deformation quantization deals with a pair of vector bundles $E^{0}, E^{1}$ over a symplectic manifold $M$ 
which are isomorphic outside a compact subset. Taking connections $\partial^{0}, \partial^{1}$ on these bundles respecting this isomorphism, we construct two deformation quantizations $Q^{0}, Q^{1}$ with coefficients in the bundles

$$
K^{0}=\operatorname{Hom}\left(E^{0}, E^{0}\right), \quad K^{1}=\operatorname{Hom}\left(E^{1}, E^{1}\right) .
$$

The trace densities $t^{0}(x, h), t^{1}(x, h)$ for these quantizations coincide outside a compact set. This allows us to define correctly the "difference of traces of the unit elements", so we set

$$
\begin{aligned}
& \text { ind }\left\{Q^{0}, Q^{1}\right\}={ }^{\prime} \operatorname{Tr} 1^{0}-\operatorname{Tr} 1^{1 "}: \\
& =\frac{1}{(2 \pi h)^{n}} \int_{M}\left(t^{0}(x, h)-t^{1}(x, h)\right) \frac{\omega^{n}}{n !} .
\end{aligned}
$$

Such a situation also arises in the theory of elliptic operators. If

$$
A: C^{\infty}\left(X, E^{0}\right) \rightarrow C^{\infty}\left(X, E^{1}\right)
$$

is elliptic, then its leading symbol $a(q, p)$ gives an isomorphism of vector bundles $\pi^{*} E^{0}$ and $\pi^{*} E^{1}$ over $M=T^{*} X$ outside a zero section $p=0$. So, again we have the problem of identification of definitions (3.24) and (3.26) and it can be easily reduced to Theorem 3.1. Indeed, consider four algebras $A_{\bar{P}_{0}}, \quad A_{P_{0}}, \quad A_{\bar{P}_{1}}, \quad A_{P_{1}}$ (using notation of Theorem 3.1). We have the isomorphisms $U_{0}, V_{0}$ between $A_{P_{0}}$ and $A_{\bar{P}_{0}}$ and $U_{1}, V_{1}$ between $A_{P_{1}}$ and $A_{\bar{P}_{1}}$ and these isomorphisms preserve traces. If $\left\{O_{i}\right\}$ denotes a locally finite coordinate covering and $\left\{\rho_{i}\right\}$ the corresponding partition of unity, we construct partition of unity $\rho_{i 0}, \quad \rho_{i 1}, \quad \bar{\rho}_{i 0}, \quad \bar{\rho}_{i 1}$ in the algebras $A_{P_{0}}, \quad A_{P_{1}}, \quad A_{\bar{P}_{0}}, A_{\bar{P}_{1}}$ respectively obtained by corresponding quantization of functions $\rho_{u}$. Then

$$
\text { ind }\left\{Q^{0}, Q^{1}\right\}=\sum_{i}\left(\operatorname{Tr} \rho_{i 0}-\operatorname{Tr} \rho_{i 0}\right)
$$

with finite number of summands and further, by Theorem 3.1

$$
\text { ind }\left\{Q^{0}, Q^{1}\right\}=\sum_{i}\left(\operatorname{Tr} \bar{\rho}_{i 0}-\operatorname{Tr} \bar{\rho}_{i 1}\right)=\text { ind }\left\{\bar{P}_{0}, \bar{P}_{1}\right\} \text {. }
$$

Thus, the result of our considerations in this section may be formulated as the following "compactness principle". 
Proposition 3.2 The index theorems for deformation quantization and, in particular, the Atiyah-Singer theorem for elliptic operators follow from the explicit formula

$$
\operatorname{Tr} 1=\int_{M} \operatorname{ch} E \widehat{A}(M) \exp \frac{\omega}{2 \pi h}
$$

for the trace of unit element for deformation quantization with non-trivial coefficients on a compact symplectic manifold.

\section{References}

[1] Bokobza-Haggiag, J.: Operateurs pseudo-differéntiel sur une varieté différentiable. Ann. Inst. Fourier, 19(1969), 125-177.

[2] Bordemann, M., Neumaier, N., Pflaum, M., Waldmann, St.: On representations of star products algebras over cotangent spaces on Hermitian line bundles. Sfb 288, Preprint No. 357, Berlin, 1998.

[3] Fedosov, B.: A simple geometrical construction of deformation quantization. J. Diff. Geom., 40(1994), 213-238.

[4] Fedosov, B.: Deformation Quantization and Index Theory. Math. Top., 9, Akademie Ferlag, Berlin (1996).

[5] Fedosov, B.: Analytic formulas for the index of elliptic operators. Trans. Moscow Math. Soc., 30(1974), 159-241.

[6] Fedosov, B.: The Atiyah-Bott-Patodi method in deformation quantization. Preprint 99/7, Uni Potsdam, 1999.

[7] Safarov, Yu.: Pseudodifferential operators and linear connections. Proc. London Math. Soc., (3)74(1997), 379-416.

[8] Voronov, T.: Quantization on supermanifolds and the analytic proof of the Atiyah-Singer index theorem. Sovrem. Problemy Matem. Noveishie Dostizh. 38, 3-118. Moscow: VINITI 1991. [English transl. in: J. Siviet Math., 64(4)(1993), 993-1069.]

[9] Widom, H.: A complete symbolic calculus for pseudodifferential operators. Bull. Sci. Math., 104(1980), 19-63. 